\title{
Le système de formation des ressources humaines en Tunisie
}

Mohamed Adel Ben Amor et Mlaoueh Ammar

\section{(2) OpenEdition}

\section{Journals}

Édition électronique

URL : https://journals.openedition.org/ries/5906

DOI : 10.4000/ries.5906

ISSN : 2261-4265

\section{Éditeur}

France Education international

\section{Édition imprimée}

Date de publication : 1 septembre 2017

Pagination : 27-34

ISBN : 978-2-85420-615-9

ISSN : 1254-4590

\section{Référence électronique}

Mohamed Adel Ben Amor et Mlaoueh Ammar, « Le système de formation des ressources humaines en Tunisie », Revue internationale d'éducation de Sèvres [En ligne], 75 | septembre 2017, mis en ligne le 01 septembre 2019, consulté le 24 juin 2021. URL : http://journals.openedition.org/ries/5906 ; DOI : https://doi.org/10.4000/ries.5906

Ce document a été généré automatiquement le 24 juin 2021

(c) Tous droits réservés 

ressources humaines en Tunisie

\author{
Mohamed Adel Ben Amor et Mlaoueh Ammar
}

\title{
Quelques données
}

1 Le dispositif de formation des ressources humaines en Tunisie comprend, depuis l'indépendance en 1956, trois systèmes : éducatif (primaire à l'école, préparatoire au collège et secondaire au lycée), professionnel (du certificat d'aptitude professionnelle au brevet de technicien supérieur) et universitaire (baccalauréat suivi de trois années et plus).

\section{Le contexte}

2 Le dispositif de formation des ressources humaines a été marqué pendant une cinquantaine d'années, par l'absence de vision globale, malgré les interconnexions entre les trois systèmes. Les systèmes éducatif et professionnel étaient longtemps interconnectés avec des filières professionnalisantes et des lycées techniques unissant l'enseignement scolaire à la formation professionnelle et technique. Les choix politiques des années 1990 ont entraîné une régression de la composante professionnelle dans le système éducatif (conversion des lycées techniques en lycées généraux, suppression de l'initiation professionnelle aux lycées, renonciation au projet du baccalauréat professionnel), si bien que le seul lien qui a persisté entre ces deux systèmes concerne l'orientation dichotomique (enseignement secondaire ou professionnel) à la fin du cycle de base (école et collège, soit 9 ans au total). Ceci marqua le début de la dépréciation du système professionnel et la massification des lycées. Par ailleurs, la seule interconnexion entre les systèmes éducatif et universitaire concerne l'orientation universitaire. Cette vision éclatée du dispositif de formation des ressources humaines en Tunisie a contribué, à partir de 2000, à la baisse du niveau des 
élèves, donc des intrants à l'université, à la dépréciation accélérée de la formation professionnelle et à la massification de l'enseignement supérieur. Les effets néfastes de cette vision, surtout la baisse du niveau des apprenants des trois systèmes, les difficultés d'insertion professionnelle et le chômage des diplômés du supérieur ont impulsé en 2012 une réflexion qui a abouti à une vision unifiée du système de développement des ressources humaines.

\begin{tabular}{|l|l|l|}
\hline Enseignement primaire & Écoles publiques & Écoles privées \\
\hline Nombre d'établissements & 4575 & 324 \\
\hline Nombre d'élèves & 1079000 (dont $48,3 \%$ de filles) & 60313 (dont $47,2 \%$ de filles) \\
\hline Nombre d'enseignants & 64944 & 5455 \\
\hline Cycle préparatoire et secondaire & & \\
\hline Nombre d'établissements & 1409 & 346 \\
\hline Nombre d'élèves & 893348 (dont $54 \%$ de filles) & 58706 (dont 34,3 \% de filles) \\
\hline Nombre d'enseignants & 75056 & 10583 \\
\hline
\end{tabular}

Source : statistiques du ministère de l'éducation, octobre 2015.

\begin{tabular}{|l|l|l|}
\hline Enseignement supérieur & Public & Privé \\
\hline $\begin{array}{l}\text { Nombre d'établissements d'enseignement } \\
\text { supérieur et de recherche }\end{array}$ & $\begin{array}{l}\text { 203, dont 31 en co-tutelle, répartis } \\
\text { sur 13 universités }\end{array}$ & 65 \\
\hline Nombre total d'étudiants & 294487 & $\begin{array}{l}30669 \\
(10,4 \%)\end{array}$ \\
\hline Nombre d'enseignants permanents & 18768 & \\
\hline Nombre de chercheurs permanents & 12515 & \\
\hline Nombre de structures de recherche & $590(309$ unités et 281 laboratoires) & \\
\hline
\end{tabular}

Source : statistiques du ministère de l'enseignement supérieur et de la recherche scientifique, juin 2016.

NB : Les statistiques exactes du ministère de la formation professionnelle et de l'emploi ne sont pas disponibles sur son site web, en raison de la mobilité permanente des apprenants vers les deux autres systèmes, ainsi que vers le marché de l'emploi. 


\section{Les défis du développement d'un système unifié de ressources humaines}

\section{Améliorer les compétences de base}

3 Comme l'attestent le classement PISA 2015 ainsi que les conclusions du Livre blanc édité par le ministère de l'Éducation en mai 2016, les élèves tunisiens présentent un déficit en compétences de base, de façon générale, surtout en sciences humaines et sociales. La marginalisation de ces compétences a débuté à la fin des années 1980, entraînant un désintérêt des institutions et des élèves, aggravé par la baisse du niveau linguistique des formateurs. La dépréciation de l'enseignement professionnel et technique a entraîné de son côté un afflux massif des élèves vers les lycées, dépassant les capacités réelles de formation des formateurs et obligeant les autorités à recruter des formateurs non qualifiés et/ou non habilités. Cette situation a été aggravée par la comptabilisation de la moyenne de l'année terminale pour $25 \%$ dans la moyenne finale au baccalauréat, sans prémunir contre les dérives abusives qui n'ont pas tardé à se manifester, affectant la crédibilité du système éducatif. La résultante est une baisse du niveau général des bacheliers, à l'entrée du système universitaire.

\section{Coordonner et articuler les trois systèmes de formation}

4 Outre les interconnexions ci-haut citées entre les trois systèmes, le dispositif de formation des ressources humaines et de développement des compétences en Tunisie doit se doter d'une vision globale et intégrée. Les passerelles entre les systèmes n'ont pas été mises en place au moment opportun, faisant du système professionnel une voie de récupération des échecs des deux autres. Certaines réformes entreprises séparément dans les trois systèmes ont aggravé leur désarticulation et occasionné des discordances.

\section{Renforcer la professionnalisation des parcours de formation}

5 La dépréciation de la formation professionnelle et la régression de l'enseignement technique et professionnel dans le système éducatif ont eu un impact négatif sur le système universitaire. De son côté, l'université n'a ni pu ni su anticiper les mutations qui font d'elle le véritable moteur du développement régional et un levier entrepreneurial. Ce clivage avec le monde socioéconomique a fait que l'université a continué à former pour des métiers à faible valeur ajoutée ou qui ont subi des mutations profondes ou même sont devenus obsolètes, de même qu'elle n'a pas anticipé l'émergence de nouveaux métiers à forte valeur ajoutée. La mise en place du système LMD, suite à l'adhésion de la Tunisie au processus de Bologne en tant que pays partenaire en 2006, n'a pas apporté les améliorations escomptées de façon notable, car sa mise en place a été déviée de ses objectifs initiaux, principalement la professionnalisation des parcours de formation en vue d'une meilleure insertion professionnelle. Les améliorations souhaitées ne se sont pas produites, entraînant un cumul de chômeurs diplômés de l'enseignement supérieur, qui représentent actuellement 38 \% de l'ensemble des chômeurs en Tunisie. 


\section{Améliorer la gouvernance des établissements et institutions}

6 Les études sur le dispositif de formation des ressources humaines en Tunisie réalisées par la Banque mondiale, l'Union européenne ou encore la Banque africaine de développement ont mis le doigt sur la mauvaise gouvernance des institutions et établissements des trois systèmes, à tous leurs niveaux hiérarchiques, qualifiée de "déficit managérial ». Si les systèmes éducatif et professionnel sont handicapés par leurs textes réglementaires, qui n'ont pas prévu les structures et mécanismes de bonne gouvernance, il en va différemment du système universitaire. Les textes qui l'organisent ont prévu, depuis la fin des années 1970, la mise en place de différentes structures de gestion administrative et académique, orientation explicitée dans la loi de $2008^{1}$ et les textes d'accompagnement. Cette loi a insisté sur la vision et les missions du système d'enseignement supérieur et de recherche, sur l'autonomie des universités grâce à un passage intermédiaire par le statut d'établissement public à caractère scientifique et technologique et sur l'assurance qualité, concepts consolidés et complétés après janvier 2011. D'après ces mêmes études, la mauvaise gouvernance des trois systèmes est principalement due à l'absence d'une démarche qualité basée sur un système de management de la qualité, ainsi qu'au faible taux d'encadrement. Ceci a amené les principaux bailleurs de fonds à recommander une clause sur la gouvernance comme critère d'acceptabilité des projets tunisiens d'appui à la réforme des systèmes éducatif, professionnel et universitaire, de même que la mise en place d'unités de gestion par objectifs, structures de management de projets, est une condition sine qua non pour la libération des fonds.

\section{Réhabiliter la formation des formateurs}

7 Durant les trente premières années d'indépendance, la formation des formateurs, dans les trois systèmes, était bien codifiée et organisée. Pour le système éducatif, les enseignants des écoles primaires étaient formés dans les instituts supérieurs de formation des maîtres et les écoles normales des instituteurs, alors que les professeurs du secondaire étaient formés dans les facultés scientifiques et littéraires, ainsi qu'à l'École normale supérieure (ENS), pour l'élite. Les enseignants professionnels et techniques, destinés aux centres de formation professionnelle et aux lycées techniques, étaient en grande partie formés à l'École normale supérieure d'enseignement technique (ENSET). Enfin, l'accès à l'enseignement supérieur était réservé aux titulaires du grade d'assistant ou plus, alors que les assistants délégués et attachés de recherche dits contractuels, ne contribuaient qu'à l'enseignement pratique, avec un plafond hebdomadaire de quatre heures d'initiation à l'enseignement.

8 Avec la dépréciation de l'enseignement professionnel et la massification du secondaire et par suite du supérieur, on a assisté à un démantèlement du dispositif de formation des formateurs, avec la suppression des instituts supérieurs de formation des maitres et des écoles normales des instituteurs, la dépréciation de l'ENS, la conversion des lycées techniques en lycées généraux et celle de l'ENSET en école d'ingénieurs. L'accès à l'enseignement primaire et secondaire fut alors ouvert à du personnel non qualifié et/ ou non habilité, alors que l'enseignement supérieur, du fait de la massification, avait recours aux contractuels et autres vacataires (surtout des professeurs de l'enseignement secondaire), qui se sont vu confier des charges horaires importantes et même des responsabilités pédagogiques. La résultante de ce démantèlement est une 
baisse notable du niveau des élèves et des étudiants, surtout dans les compétences linguistiques et gestuelles, ainsi que des formateurs, surtout sur le plan pédagogique, contribuant à l'augmentation du nombre de chômeurs diplômés du supérieur, pour la plupart difficilement employables. Ainsi, la formation des formateurs (technique, scientifique, linguistique et pédagogique) constitue actuellement le principal défi dans la réforme du dispositif de formation des ressources humaines en Tunisie.

\section{Principales réformes du dispositif de formation des ressources humaines}

\section{Mise en place de la classification nationale des qualifications (CNQ)}

9 Publiée en $2009^{2}$, la CNQ est un cadre de référence reliant les diplômes délivrés par les différentes composantes du dispositif de formation des ressources humaines aux niveaux de qualifications correspondants. La CNQ comprend sept niveaux de qualifications et six descripteurs, formulés en termes de résultats d'apprentissage, ce qui confère une meilleure visibilité aux compétences des ressources humaines tunisiennes. Néanmoins, son implémentation et sa concrétisation tardent, car elles sont tributaires de la mise en place des instances de gouvernance de ladite $\mathrm{CNQ}$, de celle des réformes des trois systèmes et de la règlementation du marché du travail, notamment la fonction publique.

\section{Coordination entre les trois systèmes de formation}

10 Depuis 2012, le rapprochement des trois systèmes a été entamé, afin de conférer au dispositif de formation des ressources humaines davantage de cohérence et de cohésion. Ceci permet de promouvoir et de mettre en valeur les qualités intrinsèques des ressources humaines dans les secteurs public et privé. Afin d'assurer une meilleure articulation des projets de réforme des trois systèmes et une meilleure coordination du dispositif de formation des ressources humaines, l'arrêté conjoint du 8 octobre 2015 a institué une commission tripartite entre les trois ministères concernés, qui statue sur les questions d'intérêt commun.

\section{L'orientation tridimensionnelle}

11 L'approche de l'orientation vers la filière de choix du candidat a été révisée à tous les niveaux d'études, dans le cadre d'une orientation tridimensionnelle élaborée par la commission tripartite en 2014. Les fondements de cette approche sont :

- Le réaménagement de l'horaire scolaire, avec allègement du volume horaire enseigné au profit des activités culturelles et sportives, de l'initiation aux métiers en situation réelle et du renforcement de l'apprentissage interactif et du numérique. Ces mesures permettront à l'élève d'améliorer ses compétences de base, de découvrir les métiers précocement et de s'initier à l'esprit entrepreneurial (portfolio compétences de l'élève).

- Au terme de l'enseignement de base, l'élève choisit parmi un panier de filières habituelles et de filières professionnelles, renonçant ainsi à l'orientation classique dichotomique (lycée vs centre de formation professionnelle), qui entraîne à la fois une massification de l'enseignement secondaire et une marginalisation de l'enseignement professionnel. En 
amont, un accompagnement par des conseillers habilités à l'orientation permet d'assister l'élève dans ses choix.

- Les nouveaux bacheliers peuvent choisir entre les filières universitaires et les filières professionnelles; depuis juin 2014, le guide de l'orientation universitaire comprend une présentation des centres de formation professionnelle et de leurs filières du niveau BTS ; le nombre de nouveaux bacheliers optant pour une filière professionnelle croît d'année en année, ce qui entraînera à moyen terme une revalorisation du système professionnel et une démassification de l'université.

- La mise en place de passerelles dans les deux sens, entre les formations professionnelle et universitaire, permet aux étudiants en cours de formation d'intégrer volontairement l'enseignement professionnel, de même qu'elle ouvre la possibilité aux titulaires du brevet de technicien supérieur (BTS) de postuler sur concours à une inscription en $3^{\mathrm{e}}$ année de licence appliquée en continuité avec le BTS. Les textes relatifs aux passerelles sont en cours de publication.

- La reprise de la réflexion sur le baccalauréat professionnel en 2015 ouvrira à ses titulaires des opportunités professionnelles à valeur ajoutée certaine, grâce au renforcement des compétences spécifiques.

- Une Instance nationale d'information et d'orientation sera créée pour concrétiser cette démarche.

\section{Gouvernance et management des ressources}

12 Le ministère de l'enseignement supérieur et de la recherche scientifique a entamé, depuis 2008, l'amélioration de la gouvernance des établissements d'enseignement supérieur et de recherche (EESR), des universités, des centres de recherche et des départements du ministère. Les conseils scientifiques, conseils d'administration, conseils des universités, cellules qualité et autres sont autant de structures de soutien à la bonne gouvernance. Néanmoins, la massification, ayant entraîné la création hâtive d'EESR sans mise à disposition adéquate des ressources surtout humaines (académiques, administratives et techniques), a freiné cet élan. À cela est venu s'ajouter le retard de création de l'Instance d'évaluation, d'assurance qualité et d'accréditation en 2013, prévue dans la loi de 2008. Le ministère a institué une commission nationale pour la réforme de l'enseignement supérieur et de la recherche scientifique en 2011, dont les travaux ont abouti à la production d'un Plan d'action stratégique (PAS) ${ }^{3}$, publié en janvier 2015. Ce dernier comprend quatre axes stratégiques: gouvernance, recherche innovation, professionnalisation des parcours de formation, vie estudiantine. Le PAS a inspiré la conception du Projet de modernisation de l'enseignement supérieur en soutien à l'employabilité (Promesse), financé par la Banque mondiale. Dans le cadre de ce projet 2016-2020, des fonds compétitifs (Projets d'appui à la qualité) ont été inscrits pour améliorer la gouvernance des EES, des universités, des centres de recherche et des départements du ministère. Des fonds spécifiques ont été également alloués pour les accompagner dans la mise en place du système de management de la qualité en vue de la certification des prestations et de l'accréditation des diplômes. La gouvernance par la qualité est ainsi l'une des actions phares du Promesse et prioritaires du ministère. 


\section{La professionnalisation des parcours de formation}

13 Depuis la promulgation de la loi de 2008, le ministère a arrêté une série de mesures dans l'objectif de renforcer l'orientation professionnalisante des parcours de formation.

- L'individualisation de licences et mastères professionnels, avec l'engagement d'une réflexion sur le doctorat professionnel.

- La mise en place d'une commission mixte entre le ministère de l'enseignement supérieur et de la recherche et l'Utica (centrale patronale) a permis un rapprochement entre l'université et le monde socio-économique, qui s'implique davantage dans l'enseignement, les stages et la recherche. Le concept d'université entrepreneuriale se développe, plaçant l'université au cœur du développement régional.

- La promulgation de la loi relative aux stages et à l'alternance, et textes y afférents, a permis de valoriser et de codifier cette activité pédagogique primordiale pour l'insertion professionnelle. Sur un autre plan, les passerelles entre les formations universitaires et professionnelles ont permis également la mise à disposition des étudiants en licence et mastère professionnels des plateformes des centres de formation professionnelle.

- La généralisation, au niveau licence, de l'enseignement de l'anglais, de l'informatique, de l'entrepreneuriat et des techniques de communication, soft skills indispensables améliorant notablement l'employabilité et la création de start-up par les jeunes diplômés.

- La mise en place, depuis 2006, de parcours (licence et mastère) co-construits entre universitaires et professionnels, a notablement amélioré l'insertion professionnelle des diplômés, en attestent les audits réalisés. Le système universitaire tunisien dispose aujourd'hui d'une expertise éprouvée en ingénierie de la co-construction, grâce à la formation de méthodologues et d'auditeurs de parcours co-construits, et à l'élaboration d'un guide méthodologique et d'un guide d'audit 5 .

- Le ministère a engagé une réflexion sur la mise en place d'un dispositif universitaire de validation des acquis de l'expérience (VAE).

- Depuis 2013, les EESR et les universités ont commencé à créer leurs propres centres de carrières et de certification des compétences (4C). Les $4 \mathrm{C}$ sont des interlocuteurs privilégiés entre l'université et l'entreprise, aident les étudiants à appréhender les métiers et leurs compétences respectives et leur facilitent l'accès aux certifications techniques et en soft skills.

\section{La formation des formateurs}

Un projet initié en 2012 et instauré en 2016, conjointement entre le ministère de l'éducation et le ministère de l'enseignement supérieur et de la recherche vise à réviser le dispositif de formation des formateurs du système éducatif. Ainsi, le projet prévoit la formation de professeurs de l'enseignement primaire, à raison de 2700 étudiants par année, dans le cadre d'une licence appliquée en éducation et enseignement, coconstruite entre les deux ministères et assurée en alternance. Neuf EESR ont été convertis en instituts supérieurs des sciences de l'éducation, dont l'accès est soumis à un test psychotechnique. Concernant l'enseignement secondaire, les deux ministères ont mis en place des mastères professionnels monodisciplinaires en alternance, reposant sur trois composantes: renforcement de la formation dans la discipline, didactique de la discipline, stage et mémoire. Ces mastères démarreront à la rentrée 2017 et recruteront parmi les titulaires de licence dans les disciplines enseignées au 
secondaire. Selon les prévisions du ministère de l'éducation, dix années transitoires seront nécessaires pour faire de ce nouveau processus la seule voie d'accès à l'enseignement primaire et secondaire.

Concernant l'enseignement supérieur, la réforme entamée dans le cadre du PROMESSE prévoit la réduction, déjà amorcée, du nombre de contractuels et autres vacataires non qualifiés, l'amélioration $d u$ processus de recrutement des enseignants, l'institutionnalisation de la formation pédagogique et autres mesures qui permettent de rehausser le niveau des enseignants universitaires. Néanmoins, le monde universitaire tunisien reste réfractaire à l'évaluation des enseignants par les pairs et par les étudiants, opposition qui sera en partie aplanie par la mise en place du système de management de la qualité.

En somme, ces mesures relatives à la formation des formateurs permettront de relever leur niveau et d'endiguer la principale lacune du dispositif de formation des ressources humaines en Tunisie.

\section{NOTES}

1. Loi ${ }^{\circ} 2008-19$ du 25 février 2008 , relative à l'enseignement supérieur.

2. Décret $n^{\circ} 2009-2139$ du 8 juillet 2009, fixant la classification nationale des qualifications.

3. https://goo.gl/c2SzQ3

4. Loi $n^{\circ} 2009-21$ du 28 avril 2009, fixant le cadre général de la formation pratique des étudiants de l'enseignement supérieur au sein des administrations, des entreprises ou des établissements publics ou privés.

5. https://goo.gl/AhfbLu

\section{INDEX}

Mots-clés : enseignement primaire, enseignement professionnel, enseignement secondaire, enseignement supérieur, formation des formateurs, système éducatif, compétences de base, cadre national de certifications, gouvernance

Palabras claves : enseñanza primaria, enseñanza profesional, enseñanza secundaria, enseñanza superior, formación de educadores de docentes, sistema educativo, competencia básica, marco nacional de cualificaciones, gobernabilidad

Index géographique : Tunisie

Keywords : primary education, vocational education, secondary education, higher education, teacher educator training, educational system, basic skills, national qualifications framework, governance 


\section{AUTEURS}

\section{MOHAMED ADEL BEN AMOR}

Mohamed Adel Ben Amor est titulaire d'une thèse ès sciences pharmaceutiques (biochimie) et du grade de professeur hospitalo-universitaire en biochimie. Il enseigne à la faculté de pharmacie de Monastir (Tunisie) et est praticien des hôpitaux. Nommé président de l'Université de Monastir (février 2011), puis directeur général de la rénovation universitaire (2011-2016) et chef du cabinet du ministre de l'enseignement supérieur et de la recherche scientifique (2014-2016) de Tunisie, il est auditeur selon la norme ISO 15189 (laboratoires d'analyses médicales) et instructeur en pédagogie universitaire. Courriel : adel.bamor2011@gmail.com

\section{MLAOUEH AMMAR}

Mlaoueh Ammar est diplômé du cycle supérieur de l'École nationale d'administration (ÉNA) et Conseiller des services publics en Tunisie. Directeur des réformes à la direction générale de la rénovation universitaire depuis 2009, il a été chargé de mission au cabinet du ministre de l'enseignement supérieur et de la recherche scientifique (2009-2016). Il est expert en matière de réforme de l'enseignement supérieur auprès du bureau national ERASMUS+ Tunisie (2014-2020). Courriel : mlaouehammar2015@gmail.com 